Théologiques

Théologiques

\title{
Tromperie et ironie
}

\section{Le cas du personnage des frères de Joseph en Gn 37}

\section{Patrick Kipasa Mayifulu}

Volume 22, numéro 2, 2014

URI : https://id.erudit.org/iderudit/1035692ar

DOI : https://doi.org/10.7202/1035692ar

Aller au sommaire du numéro

\section{Éditeur(s)}

Faculté de théologie et de sciences des religions, Université de Montréal

\section{ISSN}

1188-7109 (imprimé)

1492-1413 (numérique)

Découvrir la revue

\section{Citer cet article}

Kipasa Mayifulu, P. (2014). Tromperie et ironie : le cas du personnage des frères de Joseph en Gn 37. Théologiques, 22(2), 217-227.

https://doi.org/10.7202/1035692ar

\section{Résumé de l'article}

Cette étude est consacrée à la tromperie des frères de Joseph en Gn 37, 18-36 qui fait d'eux la cible d'une subtile ironie de la part du narrateur. Le discours du père devant la tunique ensanglantée qu'ils lui font porter (v. 33) met le lecteur dans une incertitude : le stratagème des frères réussit, mais peut-être en réalité échoue-t-il. À travers le dispositif narratif qu'il met en place, le narrateur semble envoyer quelques signaux au lecteur pour lui suggérer que la ruse des frères est éventée par leur cible, Jacob, alors que, de leur côté, ils peuvent croire qu'ils ont réussi leur coup. Une telle ironie qui ridiculise l'artifice des frères ne viserait-elle pas aussi à amener le lecteur à désapprouver leurs " valeurs " pour épouser le jugement du narrateur? 


\title{
Tromperie et ironie
}

\section{Le cas du personnage des frères de Joseph en Gn 37}

\author{
Patrick Kipasa Mayifulu* \\ Exégèse vétérotestamentaire \\ Université catholique de Louvain (Belgique)
}

\section{Introduction}

Dans la scène du complot contre Joseph en Gn 37,18-36, les frères se proposent d'agir contre leur cadet et d'induire leur père en erreur. Cette scène pleine de suspense et de rebondissements nous semble un des lieux où sont perceptibles des traits d'ironie d'une subtilité remarquable dans l'histoire de Joseph. Citons, à titre illustratif, la finesse de l'ironie qui frappe Ruben et Juda, qui révisent, chacun pour sa part, le projet collectif pour tenter de sauver Joseph de la mort. Le premier propose de le jeter vivant dans une citerne vide qu'il a choisie, sans porter la main sur lui, ce que les frères feront, sans s'apercevoir qu'ils sont en train d'être manipulés. Car l'intention de Ruben est de tirer Joseph de la citerne, à leur insu, pour le faire revenir vers son père (v. 21-22). Aux yeux de Juda, cependant, le projet de Ruben «consiste à éliminer Joseph en le laissant mourir dans son trou de manière à ne pas répandre de sang" (Wénin 2005, 67). D'où son "idée d'un crime propre" : éliminer Joseph en le vendant "sans qu'ils aient besoin de se salir eux-mêmes les mains » (Schenker 1987, 19). Pourtant, le lecteur le voit bien, en voulant sauver Joseph de la mort, Juda risque de faire échouer, sans s'en rendre compte, le projet de Ruben. La situation est particulièrement subtile: le trompeur (Ruben) est pris à son propre piège par un de ceux qu'il a réussi à abuser (Juda), sans que ce dernier sache qu'il

* Patrick Kipasa Mayifulu est doctorant à l'Université Catholique de Louvain, sous la direction d'André Wénin. Ses recherches portent sur le lien entre la parole et la violence en Gn 37-39. 
est ainsi en train de déjouer une tromperie ${ }^{1}$, ce qui naturellement renforce l'ironie. Pour finir, les deux frères sont victimes de l'ironie du narrateur: ce sont les Madianites qui réalisent pour leur propre compte, loin du regard des frères, ce que Ruben et Juda entendaient entreprendre pour sauver Joseph. Ils tirent Joseph de la citerne comme l'envisageait Ruben et ils le vendent aux Ismaélites, comme le proposait Juda.

Dans le présent essai, ce n'est pas Ruben et Juda qui nous intéresseront en particulier, c'est plutôt le personnage collectif des frères. En effet, la tromperie qu'ils mettent en œuvre dans cette scène fait d'eux de comiques victimes d'une subtile ironie de la part du narrateur. Nous nous proposons donc de voir comment cela fonctionne concrètement dans ce récit. On le devine, l'approche méthodologique qui semble convenir le mieux à l'objet de cette étude est l'analyse narrative. En effet, cette approche qui considère le texte dans son état actuel, s'attache à observer comment le narrateur (la «voix» qui raconte) met en récit l'histoire racontée pour produire sur le lecteur certains effets (Marguerat et Wénin 2012, 19). Et dans le cas qui nous concerne, elle nous permettra de voir comment le narrateur produit l'effet d'ironie aux dépens des frères.

\section{Les frères de Joseph, victimes d'une double injustice}

Dans l'exposition ${ }^{2}$ (v. 1-4), les frères sont campés par rapport au personnage de Joseph. Ils sont ceux avec qui il paît le petit bétail (v. 2b); ceux dont il est le nacar (voir Gunkel, cité par Skinner 1956², 444; Westermann 1986, 36) c'est-à-dire le plus jeune mais aussi le serviteur (v. 2c); mais également ceux dont Joseph parle à son père, en lui rapportant des choses qu'il présente comme méchantes (v. 2d) (voir Fokkelman 1996, 156; Wenham 1994, 350; Schenker 1987, 14)33. Ce comportement de Joseph

1. Pour pouvoir délivrer Joseph, il fallait bien que Ruben cache l'essentiel de son plan aux frères. Pourtant, c'est précisément parce que Juda en ignore le mobile qu'il peut avancer sa proposition. Or, paradoxalement, la proposition de Juda risque de faire échouer le plan de Ruben. C'est pour cela que celui-ci est le premier à aller à la citerne pour la trouver désespérément vide: il y est allé avant que - ou de peur que - les autres ne mettent à exécution le plan de Juda.

2. C'est un moment de l'intrigue que Ska $(2011,23)$ définit comme «la présentation des informations indispensables sur l'état des faits, qui précède le début de l'action "; l'exposition "précise le qui, le quoi (et parfois) le comment» (Marguerat et Bourquin $\left.2009^{4}, 59\right)$.

3. Ces auteurs estiment que Joseph est un véritable informateur qui fabriquerait luimême des propos négatifs sur ses aînés. 
s'expliquerait, peut-être, par ce que le narrateur révèle ensuite, à savoir la préférence de Jacob pour lui (Wenham 1994, 350). La raison en est aussitôt précisée: «il était pour lui un fils de vieillesse $\mathrm{e}^{5}$ ( $v$. 3b). La tunique d'apparat qu'il lui fait manifeste évidemment cette préférence, exprime la position privilégiée que le père lui accorde dans la fratrie, et l'investit de

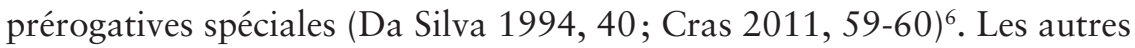
frères voient à travers cette tunique que "c'est lui [Joseph] qu'aimait leur père plus que tous ses frères» (v. $4 \mathrm{a})$. Au fond, cette tunique les fait souffrir : ils se voient comme les frères de Joseph; mais dans le regard du père, ils se sentent dévalorisés. En effet, "puisque le terme "frères", écrit Wénin, rappelle ce qui les rend égaux à Joseph, l'inégalité de traitement de la part de "leur père" à tous apparaît comme une injustice, une injure à la fraternité » (Wénin 2005, 33). Ce qui les conduit à la haine qui affecte d'abord la parole et la paix (ils ne peuvent parler à Joseph en paix, v. 4b). Elle est certes orientée vers Joseph qui fait obstruction à leur désir; toutefois, elle atteint aussi, bien qu'indirectement, le père qui étale outrageusement son amour préférentiel pour le jeune frère (voir Westermann 1987; 1996, 5-6; Turner 2000, 159; Hamilton 1995, 409).

Remarquons que dans la scène où l'action se noue (v. 5-11), la haine des frères par laquelle le narrateur clôture l'exposition connaît une courbe ascendante que mettent en évidence ses insistances: «ils le haïrent» (v. 4), "et ils ajoutèrent encore à le haïr " (v. 5b), «et ils ajoutèrent encore à le haïr» (v. 8b). Le ressentiment des frères s'accroît chaque fois que Joseph prend la parole; et il la prend précisément pour leur faire le récit de ses rêves (v. 6-7.9), ce qui ne fait que les exaspérer encore davantage. Après la réaction énergique du père au récit du deuxième rêve (v. 10), le narrateur note qu' «ils le jalousèrent» (v. 11a). Ce qui ne manquera pas de surprendre le lecteur, c'est que le jeune frère semble insensible aux réactions

4. Wenham rappelle en effet que ce favoritisme est une longue et vieille histoire dans la famille de Jacob. Isaac aimait Esaü plus que Jacob, Rebecca aimait Jacob plus qu'Esaü; Jacob préférait Rachel à Léa (Gn 25,28; 29,30). Et son vieil amour pour Rachel, le voilà transféré à Joseph, le fils de celle-ci. Il n'est pas étonnant que ses frères le haïssent. Le narrateur, à trois reprises (v. 4.5.8), enregistre bien l'intensification de leur sentiment.

5. Toutes les traductions du texte hébreu sont nôtres. Elles sont volontairement littérales, voire inélégantes, pour bien mettre en évidence les subtilités de la langue hébraïque qu'un français châtié ne réussit pas toujours à rendre fidèlement.

6. Pour Cras (2011, 59-60) «c'est de l'amour paternel dont Joseph est revêtu de façon princière [...]. C'est l'insigne d'un privilège et d'une haute dignité qui le place incontestablement au-dessus de ses frères". 
de plus en plus haineuses de ses aînés. Ses initiatives de dialogue sont au mieux de l'ingénuité ou de la bonne volonté mal placée, au pire de la fanfaronnade, voire de la provocation.

Ainsi, dès l'amorce de l'histoire et de façon plus ou moins subtile, le lecteur comprend que Joseph est caractérisé comme un frère dont la parole ne semble pas de nature à favoriser la fraternité et la paix, tandis que les frères sont introduits comme les victimes d'une double injustice: de la part de Joseph qui, sans doute à leur insu, parle d'eux en mal (v. 2), leur raconte à trois reprises ${ }^{7}$ des rêves qui, à leurs yeux, le destinent à un avenir de pouvoir (v. 5-11), et du père qui leur préfère ce frère qui, par ailleurs, est le serviteur des fils des servantes (v. 2) et qui, en outre, les prive de l'amour paternel.

\section{Les frères s'éloignent de Joseph et de Jacob (v. 12-14)}

À la fin de la scène des rêves, les frères s'éloignent de ceux auprès de qui ils ne semblent plus avoir aucune place. Ils s'en vont paître le bétail à Sichem (v. 12). Israël envoie Joseph vers eux, chargé d'une mission. Le lecteur en connaît l'objet: littéralement, lui faire "revenir une parole au sujet de la paix des frères et de la paix du troupeau » (v. 14); par ailleurs, il a lu le v. 4 où il est déjà question de shalôm à propos de la parole, et le v. 2 où Joseph «fait venir une parole» mais qui est de «mal». Il dispose donc d'une clé supplémentaire qui lui permet d'entendre autrement cette mission confiée à Joseph. On a ici une sorte d'ironie: le lecteur qui en sait plus que Jacob comprend en effet que ce qu'il fait, sans en être complètement conscient, est particulièrement adapté à la situation; car il vise exactement ce qui fait mal, à savoir l'unité de la fratrie.

Cette position supérieure, en outre, permet au lecteur de prendre la mesure du risque que Jacob fait courir à Joseph en le laissant aller tout seul au loin vers des personnes qui le détestent et le jalousent. Il y a de quoi avoir peur pour Joseph et se demander comment cela va se terminer.

\section{Tromperie et ironie}

La scène du complot des frères contre le jeune Joseph commence par une intervention du narrateur qui, recourant à sa faculté d'omniscience,

7. Il convient de rappeler que Joseph a bel et bien eu deux rêves. Mais il a fait trois récits de rêves. Le premier, il le conte aux frères (v. 5); le second, il le raconte d'abord aux frères seuls (v. 9), puis aux frères et au père en même temps (v. 10). 
informe le lecteur du complot des frères contre Joseph (v. 18). Aussitôt après, les personnages eux-mêmes expriment clairement ce qu'ils projettent de faire et la façon dont ils entendent s'y prendre concrètement: ils prévoient de «tuer» Joseph, de le «jeter» dans une des citernes et de «dire» qu' "une bête méchante l'a mangé ». Ainsi ses rêves seront démentis. Il y a ici une ellipse intéressante, car les frères ne précisent pas ce qu'ils feront concrètement pour rendre une telle version crédible, ils ne parlent pas de la preuve matérielle qui servira à corroborer un tel discours. Le lecteur s'aperçoit qu'en réalité, l'intention des frères est d'agir de manière à ne pas se faire découvrir. D'où la double dissimulation derrière laquelle ils se proposent de cacher leur crime, à savoir : celle du corps (en le jetant dans une des citernes) et celle de la vérité (en inventant une fable; voir Wénin 2005, 59-60). Leur stratégie de dissimulation réussira-t-elle? C’est ce que l'on va voir. Mais le lecteur comprend aussi que Jacob est la cible de la mystification qu'ils sont en train de mettre en place et dont il observera le déploiement plus tard (v. 31-33).

Lorsque Joseph arrive près de ses frères, ceux-ci commencent par une action qui ne figure pas dans le complot initial, à savoir le traitement réservé à la tunique. C'est une surprise pour le lecteur qui comprend du même coup que c'est à cette tunique qu'ils ont reconnu Joseph tandis qu'il était encore loin. Le narrateur insiste sur cette tunique, s'y attarde en l'assortissant de précisions. Il envoie ainsi un signal au lecteur. Il veut lui montrer qu'avant de mettre leur plan à exécution, les frères commencent par s'en prendre à ce vêtement, première cause de leur animosité contre Joseph (voir Green 1996, 48), signe de l'amour du père qui confère à leur petit frère un statut spécial et provoque leur haine. En l'en dépouillant, les frères privent Joseph de la marque de sa supériorité qui nourrit leur haine (cf. v. 3-4). À voir ce geste, cependant, le lecteur comprend que Joseph est en même temps aussi libéré «de ce qui reste de l'emprise de son père sur lui. S'il en est ainsi, ôter la tunique, c'est paradoxalement faire un pas en direction de la fraternité, et donc aussi de la réalisation de ce que, depuis les versets 13-17, le lecteur sait être le désir profond d'Israël et celui de Joseph» (Wénin 2005, 65).

La tunique une fois enlevée, les frères agissent conformément au plan de Ruben dont ils ignorent pourtant qu'il procède d'une manipulation dont ils sont l'objet (v. 21-22). Joseph est jeté dans la citerne que leur aîné a désignée. Quoi qu'il en soit, dans l'Ancien Testament, une citerne vide, sans eau, est un lieu symbolique de la mort, d'une mort qu'on dissimule (voir Da Silva 1994, 80-81; Cohen 2012, 67). Mais entre-temps, à l'insu 
des frères, les marchands madianites, qui passent par-là, tirent Joseph et le vendent aux Ismaélites contre vingt pièces d'argent (v. 28).

Une fois que Ruben les a mis au courant de la disparition de Joseph, les frères s'occupent, sans état d'âme, de régler le problème avec leur père. Le lecteur peut alors voir comment ils vont concrètement mettre en place le stratagème envisagé dès la scène du complot (v. 19): ils trempent la tunique dans le sang d'un bouc qu'ils ont égorgé; ils font porter la tunique maculée de sang vers leur père, l'invitant à se prononcer: est-ce la tunique de Joseph ou non? On le voit, la mention de la tunique ensanglantée comble l'ellipse du v. 20b ("et nous dirons: "une bête méchante l'a mangé" »), car c'est ce vêtement qui constitue la pièce à conviction susceptible de corroborer l'idée de sa mort violente.

La manière dont les frères procèdent amène le lecteur à déduire leur intention: pour avertir le père qu'un malheur est arrivé à Joseph, n'utilisent-ils pas la tunique afin de le punir au moyen de ce par quoi il les a fait souffrir? (voir Green 1996, 52-53). C'est bien possible. Et quand Jacob reconnaît la tunique, il arrive exactement - au mot près - à la conclusion que les frères avaient imaginée au point de départ: "une bête méchante l'a mangé» (v. 20 et v. 33). Le lecteur dispose d'un certain nombre de clés qui lui permettent de comprendre qu'au point de départ, les frères avaient une idée lorsqu'ils tramaient leur complot (v. 19-20); ils ont mis en place des conditions pour que le père tire la conclusion qu'ils voulaient. Et sa répartie indique qu'il s'est laissé prendre au piège, puisqu'il arrive effectivement à cette conclusion. Le narrataire devine donc que le stratagème mis au point réussit parfaitement aux dépens de Jacob qui reste en position inférieure et semble ne pas se rendre compte qu'il a été dupé. La position supérieure dont le lecteur est crédité lui permet de faire cette déduction. Car il a entendu, et les frères (v. 19-20), et Jacob (v. 33); mais les frères n'ont pas entendu Jacob, ni Jacob, ses fils.

Lorsque le père parle (cf. v. 33), le lecteur sait qu'en réalité la bête qui a mangé Joseph, c'est la méchanceté des frères. Il n'a pas oublié la longue expérience de mensonges et de dissimulations de Jacob. Il sait bien qu'autrefois, Jacob a piégé son vieux père au moyen du vêtement d'Esaü, le fils chéri, afin d'hériter de la bénédiction paternelle destinée à ce dernier ${ }^{8}$. Le lecteur peut se demander si le père n'est pas intrigué par cette tunique qui

8. Il y a une belle ironie ici aux dépens de Jacob : le voilà à présent séparé de son fils préféré par les autres qui usent d'un artifice semblable à celui auquel il a recouru jadis, afin de jouir eux aussi de l'amour paternel. 
lui revient pleine de sang, sans qu'il voie la dépouille même de Joseph; ne lui rappelle-t-elle pas sa propre histoire? N'y flaire-t-il pas quelque chose de la ruse de ses fils? (voir Eisenberg et Gross 1983, 169; Wénin 2005, 81). Le lecteur est bien capable d'avoir accès au second sens de ce que dit Jacob sans être sûr que Jacob en est lui-même conscient. Il se trouve ainsi dans une situation d'incertitude qui aiguise sa curiosité: le stratagème des frères réussit, mais peut-être échoue-t-il, en réalité. Et s'il s'avérait que Jacob, que les fils ont voulu tromper, ait démasqué à leur insu leur initiative de dissimulation, cela signifierait que le trompé a pris l'avantage sur les trompeurs (ses fils). L'ironie serait alors maximale, le lecteur s'apercevant que la ruse est éventée par la cible alors que les trompeurs, de leur côté, croient avoir réussi leur coup (Marguerat et Wénin 2012, 279).

Le fait que le père conclue que Joseph est mort mais qu'il refuse de «se laisser consoler ", pourrait laisser entendre qu'il subodore qu'on lui cache quelque chose. Le lecteur ne peut que soupçonner cela, sans avoir des bases solides pour le fonder. Par contre, lorsqu'il avancera dans le récit, il verra clairement que Jacob se méfie de ses fils parce qu'il a compris qu'ils sont pour quelque chose dans la disparition de leurs frères, Joseph et Siméon (Gn 42,4.36). C'est alors que le lecteur aura la certitude qu'en réalité, le double sens des paroles de Jacob (v. 33) était voulu.

Mais l'initiative du message que les frères font porter au père ne vise pas qu'à le faire souffrir. Ils espèrent sans doute obtenir aussi — mais sans le révéler clairement - l'harmonisation des relations au sein de la famille, la constitution du cercle familial autour d'un père désormais sans préféré, pour jouir enfin de l'amour paternel dont le frère disparu les éloignait (voir Schenker, 1987, 21; Marx 2002, 112). C'est ainsi qu'ils tentent de consoler leur père (v. 35). Mais l'affliction de Jacob est telle qu'on pourrait le croire père d'un fils unique qui ne trouve aucune consolation dans les autres fils (voir Josèphe 2003, 185). Sa souffrance semble davantage l'éloigner de ceux qu'il pourrait même soupçonner d'être impliqués dans la disparition de Joseph. Pas de réconciliation ni de paix véritables, là où il y a "déni de l'autre " (Wénin 2005, 82), mensonge et duplicité. Leur "espoir de réconciliation» (Wénin, 2004, 407-410) est déçu. Sur ce point précis, ironiquement, le subterfuge semble avoir produit le contraire des effets escomptés: il se serait retourné contre ses auteurs. Ce qui pourrait faire sourire le lecteur.

Il serait intéressant d'observer qu'en plus du sourire qu'elle arrache au lecteur, cette ironie qui frappe les frères a également une autre fonction. Le lecteur se souvient que dans les deux premières scènes de Gn 37, les frères 
sont campés comme les victimes d'injustice de la part du père et de Joseph. Une telle présentation contribue à générer dans l'esprit du lecteur un sentiment de sympathie pour eux. Si dans les deux premières scènes, leur haine pouvait encore être justifiable ou compréhensible, dans la scène du complot, en revanche, leur désir de vengeance, leur volonté de dissimulation et de tromperie ainsi que leur violence - autant de contre-valeurs que le récit met en évidence - induisent chez le lecteur un sentiment de répulsion. Il n'a guère envie de s'identifier à eux ou de les prendre en sympathie. On se souviendra que l'ironie est de "nature évaluative» (Schoentjes 2001, 145) En ironisant ainsi sur l'échec des projets des frères qui, en plus d'être introduits dans cette scène (v. 18-35) comme des méchants, sont construits, avec une certaine drôlerie, comme des personnages qui n'ont que peu de prise sur ce qui leur arrive, même au moment où ils croient pouvoir reprendre les choses en main et les retourner en leur faveur, le narrateur frappe de discrédit leur système de valeurs. L'ironie dont ils sont la cible aurait donc aussi une fonction axiologique. Car elle amène le lecteur à désapprouver les «valeurs" des frères, pour épouser «le point de vue évaluatif du narrateur» (Marguerat et Bourquin 20094, 155), c'est-à-dire le «jugement du narrateur induisant sa présentation des personnages ou des choses, en fonction de son système de valeurs et de sa conception du monde» (Marguerat et Bourquin 20094, 231).

\section{Conclusion}

La tromperie que les frères mettent en œuvre dans la scène que nous venons d'examiner fait d'eux, en fin de compte, la cible de l'ironie que ménage la narration. Leur stratagème ne produit pas totalement le résultat escompté. Le mal qu'ils cherchaient, à savoir faire souffrir le père pour lui faire payer l'humiliation qu'il leur a fait endurer (voir Wénin 2004; 2005, 76) se réalise: il déchire ses vêtements, met un sac sur ses reins, prend un long deuil et repousse toute consolation. En revanche, le bien qu'ils voulaient, à savoir l'harmonisation de relations au sein de la famille, ne se concrétise pas. Il est intéressant de remarquer qu'un seul des deux buts est atteint et c'est ironiquement celui qui fait mal, casse les relations et empêche l'harmonisation désirée. Car, à présent, le père semble davantage

9. Parlant de l'ironie verbale, Schoentjes précise encore davantage cette nature évaluative de l'ironie quand il écrit: "l'ironie est toujours un jugement de valeur. La plaisanterie juge en effet celui qu'elle prend pour cible du ridicule et si le public rit, il confirme le jugement de l'orateur» $(2001,81)$. 
éloigné d'eux qu'il ne l'était avant la disparition de Joseph. Autant dire que les bourreaux (les frères) se trouvent désormais en face d'une victime (le père) dont la souffrance leur reproche en permanence leur forfait collectif.

\section{Références}

Cohen, N. J. (2012), Masking and Unmasking Ourselves. Interpreting Biblical Texts on Clothing \& Identity, Woodstock, Jewish Lights Pub.

Cras, A. (2011), La symbolique du vêtement dans la Bible. Pour une théologie du vêtement, Paris, Cerf.

DA Silva, A. (1994), La symbolique des rêves et des vêtements dans l'histoire de Joseph et de ses frères, Québec, Fides (Héritage et projet 52).

Eisenberg, J. et Gross, B. (I983), Un Messie nommé Joseph, Paris, Albin Michel (Présence du Judaïsme).

Forkelman, J. (1996), "Genesis 37 and 38 at the Interface of Structural Analysis and Hermeneutics", dans L. J. DE REGT et al., dir., Literary Structures and Rhetorical Strategies in the Hebrew Bible, Assen, Van Gorcum/Eisenbrauns, p. 152-187.

Green, B. (1996), "What Profit for Us? » Remembering the Story of Joseph, Lanham/New York/London, University Press of America.

Hamilton, V. P. (1995), The Book of Genesis. Chapters 18-50, Grand Rapids, Eerdmans (New International Commentary on the O.T.).

JosèPhe, F. $\left(2003^{3}\right)$, Les Antiquités juives, Livre I / texte, trad. et notes par E. Nodet, G. Berceville et E. Warschavski, Paris, Cerf.

Marguerat, D. et Bourquin, Y. (20094), Pour lire les récits bibliques. Initiation à l'analyse narrative, Paris/Genève, Cerf/Labor et Fides.

Marguerat, D. et Wénin, A. (2012), Saveurs du récit biblique, Genève/ Montrouge, Labor et Fides/Bayard.

Marx, A. (2002), «Le rituel biblique du Yom hakkipourîm (Lévitique 16) », dans S. Trigano, dir., La Bible et l'autre, Paris, In Press (Pardès. Études et culture juives 32-33), p. 106-115.

SCHenker, A. (1987), Chemins bibliques de la non-violence, Chambéry, C.L.D.

SKA, J.-L. (2011), "Nos pères nous ont raconté». Introduction à l'analyse des récits de l'Ancien Testament, Paris, Cerf (Cahiers Evangile 155).

Skinner, J. (1956²), A Critical and Exegetical Commentary on Genesis, Edinburg, T\&T Clark (ICC). 
Schoentjes, P. (200I), Poétique de l'ironie, Paris, Seuil (Points essais 467).

Turner, L. A. (2000), Genesis, Sheffield, Academic Press (Readings: A New Biblical Commentary).

Wenham, G. J. (1994), Genesis 16-50, Dallas, Word Books (Word Biblical Commentary 2).

WéNIN, A. (2004), «La tunique ensanglantée de Joseph (Gn 37, 31-33): un espoir de réconciliation? ", dans Vetus Testamentum, 54, p. 407-410.

(2005), Joseph ou l'invention de la fraternité. Lecture narrative et anthropologique de Genèse 37-50, Bruxelles, Lessius (Le livre et le rouleau 21).

Westermann, C. (1986), Genesis 37-50. A Commentary, Minneapolis, Augsburg Publishing House. Eerdmans.

(1987), Genesis. A Practical commentary, Grand Rapids, (1996), Joseph. Studies of the Joseph Story in the Genesis, Edinburgh, T\&T Clark.

\section{Résumé}

Cette étude est consacrée à la tromperie des frères de Joseph en Gn 37, 18-36 qui fait d'eux la cible d'une subtile ironie de la part du narrateur. Le discours du père devant la tunique ensanglantée qu'ils lui font porter (v. 33) met le lecteur dans une incertitude: le stratagème des frères réussit, mais peut-être en réalité échoue-t-il. À travers le dispositif narratif qu'il met en place, le narrateur semble envoyer quelques signaux au lecteur pour lui suggérer que la ruse des frères est éventée par leur cible, Jacob, alors que, de leur côté, ils peuvent croire qu'ils ont réussi leur coup. Une telle ironie qui ridiculise l'artifice des frères ne viserait-elle pas aussi à amener le lecteur à désapprouver leurs «valeurs» pour épouser le jugement du narrateur?

\section{Abstract}

This study is devoted to the deception carried out by Joseph's brothers in Genesis 37, 18-36, which makes them the target of the narrator's subtle irony. The father's speech before the bloody tunic brought to bim (v. 33) casts some doubt on the reader's mind: the stratagem devised by the brothers apparently succeeds - but perhaps it actually fails. Through the narrative device used, the narrator seems to send signals to the reader suggesting that 
the brothers' trick is revealed by their target, Jacob, while they may themselves believe their ruse has succeeded. Does such an irony mocking the artifice of the brothers not lead the reader to disapprove their "values" and endorse the narrator's judgment? 